Pacific Journal of Mathematics

KRULL RINGS 


\section{KRULL RINGS}

\section{ROBERT E. KENNEDY}

We extend the notion of a Krull domain to commutative rings with identity which may contain zero divisors. In order to do this we present a definition of the divisors of an arbitrary ring, and show that the collection of divisors is a commutative semigroup with identity and is a group if and only if the ring is completely integrally closed. In addition, an extension of unique factorization domains to arbitrary commutative rings is used to investigate the relationship between Krull rings and unique factorization rings. In particular, it is shown that a unique factorization ring is a Krull ring with trivial class group.

1. Divisors and complete integral closure. The divisors of an integral domain together with the concept of complete integral closure is important in the study of unique factorization domains and Krull domains. To extend the notion of divisors to rings which contain zero divisors, let $R$ be a ring with total quotient $\operatorname{ring} K$. If $A$ is a subset of $K$ such that $A$ is an $R$-module and there exists a regular element $d \in R$ where $d A$ is a subset of $R$, then $A$ is called a fractional ideal of $R$. If, in addition, $A$ contains a regular element of $R$, then $A$ is called a regular fractional ideal of $R$. In what follows, $F(R)$ will denote the collection of regular fractional ideals of $R$.

For $A, B \in F(R)$, set $[A: B]=\{x \in K \mid x B \subset A\}$. Then $[A: B] \in F(R)$. In particular, $[R: A] \in F(R)$ for each $A \in F(R)$. Define $\sim$ on $F(R)$ by: $A \sim B$ if and only if $[R: A]=[R: B]$. Note that $\sim$ is an equivalence relation on $F(R) . \quad D(R)$ will denote the collection of equivalence classes induced on $F(R)$ by $\sim$. If $A \in F(R)$, then $\operatorname{div} A$ represents the equivalence class containing $A$. As in the domain case, a well-defined operation may be defined on $D(R)$ by: $\operatorname{div} A+\operatorname{div} B=\operatorname{div} A B$ for $A, B \in$ $F(R)$. With this operation, $D(R)$ is a commutative semigroup with identity $\operatorname{div} R$. If $D(R)$ is a group, then $D(R)$ is called the group of divisors of $R$.

For $A \in F(R)$, set $\bar{A}=[R:[R: A]]$. Then $\bar{A} \in F(R)$ and $A \sim \bar{A}$. In fact, for $A, B \in F(R), A \sim B$ if and only if $\bar{A}=\bar{B}$. A partial order may be defined on $D(R)$ by: $\operatorname{div} A \leqq \operatorname{div} B$ if and only if $\bar{B} \subset \bar{A}$. With this partial order, $D(R)$ is a partially ordered semigroup with identity.

The following proposition is of importance since it gives a necessary and sufficient condition for a ring to be completely integrally closed.

Proposition 1.1. Let $R$ be a ring with total quotient ring $K$. 
Then $R$ is completely integrally closed if and only if $D(R)$ is group.

Proof. Suppose that $R$ is completely integrally closed. Let $A \in F(R)$. It only needs to be shown that $\operatorname{div} A$ has an additive inverse in $D(R)$.

Let $x \in[R: A[R: A]]$. Then $x[R: A] \subset[R: A]$. Therefore $x^{n} A[R: A] \subset$ $A[R: A] \subset R$ for each positive integer $n$. Let $d$ and $d^{\prime}$ be regular elements of $R$ which are contained in $A$ and $[R: A]$ respectively. Hence $d d^{\prime}$ is regular and $d d^{\prime} x^{n} \in R$ for each positive integer $n$. Thus by complete integral closure, $[R: A[R: A]] \subset R$. But then it follows that $[R: A[R: A]]=R$ and so $\operatorname{div} R=\operatorname{div} A+\operatorname{div}[R: A]$, and $\operatorname{div}[R: A]$ is the additive inverse of $\operatorname{div} A$ in $D(R)$.

The proof of the converse is similar to the analogous theorem in the domain case.

An immediate corollary to the above proposition is that if $R$ is completely integrally closed, then $D(R)$ is a lattice ordered abelian group. In fact if $\operatorname{div} A$ and $\operatorname{div} B$ are elements of $D(R)$, it follows that $g l b\{\operatorname{div} A, \operatorname{div} B\}=\operatorname{div}(A+B)$ and lub $\{\operatorname{div} A, \operatorname{div} B\}=\operatorname{div}(A \cap B)$.

A regular fractional ideal of a ring $R$ is called a principal fractional ideal if it is of the form $R a$ where $a$ is a regular element of the total quotient ring of $R$. It can be shown that $\bar{A}$ is equal to the intersection of all principal fractional ideals which contain $A$ for each regular fractional ideal $A$ of a ring $R$. If $A=\bar{A}$, then $A$ is said to be a divisorial ideal of $R$.

2. Krull rings. Let $R$ be a ring with total quotient ring $K$ such that $R \neq K$. Then $R$ is called a Krull ring if there exist a family $\left\{\left(V_{\alpha}, P_{\alpha}\right) \mid \alpha \in I\right\}$ of discrete rank one valuation pairs of $K$ with associated valuations $\left\{V_{\alpha} \mid \alpha \in I\right\}$ such that

(I) $R=\bigcap\left\{V_{\alpha} \mid \alpha \in I\right\}$.

(II) $v_{\alpha}(a)=0$ almost everywhere on $I$ for each regular $a \in K$, and each $P_{\alpha}$ is regular ideal of $V_{\alpha}$.

Note that for $P_{\alpha}$ to be a regular ideal of $V_{\alpha}$, it is necessary and sufficient that $P_{\alpha}$ contain a regular element of $R$. The last condition also means that for each regular element $a \in R, a$ is an element of $V_{\alpha}-P_{\alpha}$ for all except finitely many $\alpha \in I$.

For $A \in F(R), \inf \left\{v_{\alpha}(a) \mid a \in A\right\}$ exists for each $\alpha \in I$ since the value of a regular element is finite. For $\alpha \in I, v_{\alpha}(A)$ will denote this infinum.

Proposition 2.1. Let $R$ be a Krull ring with a defining family, $\left\{v_{\alpha} \mid \alpha \in I\right\}$, of discrete rank one valuations on $K$ where $K$ is the total quotient ring of $R$. 
(i) Let $A, B \in F(R)$ where $B$ is divisorial. Then $A \subset B$ if and only if $v_{\alpha}(A) \geqq v_{\alpha}(B)$ for each $\alpha \in I$.

(ii) For each $A \in F(R), v_{\alpha}(A)=0$ almost everywhere on $I$.

Proof. The proof is similar to the analogous theorem in the domain case.

Proposition 2.2. Let $R$ be a Krull ring with total quotient ring $K$. Then $R$ is completely integrally closed and every nonempty set of divisorial ideals of $R$ has a maximal element.

Proof. Let $\left\{v_{\alpha} \mid \alpha \in I\right\}$ be a defining family of discrete rank one valuations on $K$. Let $d$ be a regular element in $R$ and $x \in K$ such that $d x^{n} \in R$ for each positive integer $n$. Hence $v_{\alpha}(d)+n v_{\alpha}(x) \geqq 0$ for each positive integer $n$. Suppose that $x \in K-R$. Then there exists $\beta \in I$ such that $v_{\beta}(x)<0$. Since $d$ is fixed, $n$ may be chosen large enough so that $v_{\beta}(d)+n v_{\beta}(x)$ is strictly less than zero, a contradiction. Hence $v_{\alpha}(x) \geqq 0$ for each $\alpha \in I$, and $x \in R$. Therefore, $R$ is completely integrally closed.

That each nonempty set of divisorial ideals of $R$ has a maximal element follows from Proposition 2.1.

Let $R$ be a Krull ring. Then by Proposition 2.2, every nonempty collection of positive elements of $D(R)$ has a minimal element. Let the set of all minimal positive elements of $D(R)$ be indexed by a set I. For each $\alpha \in I$, let $M_{\alpha}$ be the divisorial ideal of $R$ such that $\operatorname{div} M_{\alpha}$ is a minimal positive element of $D(R)$. Thus $\left\{M_{\alpha} \mid \alpha \in I\right\}$ is the collection of maximal divisorial ideals of $R$. The proof of the following lemma is omitted since its proof is similar to that of the domain case.

LeMma 2.3. Let $R$ be a completely integrally closed ring with maximal divisorial ideal $M$. If $\operatorname{div} M \leqq \operatorname{div} A_{1}+\operatorname{div} A_{2}+\cdots+\operatorname{div} A_{n}$, where each $\operatorname{div} A_{i}$ is a positive element of $D(R)$, then $\operatorname{div} M \leqq \operatorname{div} A_{i}$ for some $i$.

Proposition 2.4. Let $R$ be a Krull ring. Using the notation preceding the lemma, each element of $D(R)$ is uniquely of the form

$$
\sum n_{\alpha} \operatorname{div} M_{\alpha}
$$

where $n_{\alpha}=0$ almost everywhere on $I$.

Proof. Let $\operatorname{div} A \in D(R)$. By the corollary to Proposition 1.1, there exists $B \in F(R)$ such that $\operatorname{div} B$ is the least upper bound of 
$\{\operatorname{div} A, \operatorname{div} R\} . \quad$ Then, since $D(R)$ is an abelian group, $\operatorname{div} A=\operatorname{div} B$ ( $\operatorname{div} B-\operatorname{div} A$ ) and each element of $D(R)$ may be written as the difference of two nonnegative elements of $D(R)$. Thus, if it is shown that each positive element of $D(R)$ is of the desired form, then so is every element of $D(R)$. That this is indeed the case is straight forward. Uniqueness of the above representation follows from Lemma 2.3.

It can be shown that a finite complete direct sum of Krull rings is a Krull ring. However, it is not necessary that each summand of a complete direct sum be Krull in order that it be a Krull ring. The following proposition demonstrates this possibility.

Proposition 2.5. Let $R$ be a Krull ring with total quotient ring $K$, and $S$ be a ring which is its own total quotient ring. Then $R \oplus S$ is a Krull ring.

Proof. Let $\left\{\left(V_{\alpha}, P_{\alpha}\right) \mid \alpha \in I\right\}$ be a defining family of discrete rank one valuation pairs of $K$ for $R$. Note that the total quotient ring of $R \oplus S$ is $K \oplus S$. For each $\alpha \in I$, define $w_{\alpha}(x, s)=v_{\alpha}(x)$ where $v_{\alpha}$ is a determining valuation on $K$ for $\left(V_{\alpha}, P_{\alpha}\right)$. Then each $w_{\alpha}$ is a discrete rank one valuation on $K \oplus S$.

Let $W_{\alpha}=\left\{(x, s) \in K \oplus S \mid w_{\alpha}(x, s) \geqq 0\right\}$. Then $W_{\alpha}=W_{\alpha} \oplus S$ and

$$
\bigcap\left\{W_{\alpha} \mid \alpha \in I\right\}=R \oplus S .
$$

Since $w_{\alpha}(x, s)=0$ almost everywhere on $I$ for each regular element $(x, s) \in K \oplus S$, it follows that $R \oplus S$ is a Krull ring.

3. Unique factorization rings. Let $R$ be a ring with total quotient ring $K$ such that $R \neq K . H(R)$ will denote the subgroup of $D(R)$ whose elements are of the form $\operatorname{div} R a$ where $a$ is a regular element of $K$. The factor group $C(R)=D(R) / H(R)$ is called the divisor class group of $R$.

In the domain case, $R$ is a unique factorization domain if and only if $R$ is a Krull ring and $C(R)$ is trivial. To investigate what happens when $R$ is an arbitrary ring with identity, a characterization of a unique factorization ring will be used. This characterization states that every unique factorization ring is a finite complete direct sum of unique factorization domains and of special principal ideal rings (1).

In connection with the following proposition, a ring $R$ is said to have Property $(M)$ if each nonempty collection of regular principal ideals of $R$ contains a maximal element. 
quotient ring. Consider the following statements:

(1) $R$ is a unique factorization ring.

(2) $R$ is a Krull ring and $C(R)$ is trivial.

(3) $R$ is a Krull ring and each maximal divisorial ideal of $R$ is principal.

(4) $R$ has property ( $M$ ) and the intersection of two regular principal ideals is principal.

(5) $R$ is a Krull ring and the intersection of two regular principal ideals is principal.

Then $(1) \Rightarrow(3) \Leftrightarrow(5) \Leftrightarrow(2) \Rightarrow(4)$.

Proof. $\quad(2) \Leftrightarrow(3)$. It is clear that $(2) \Rightarrow(3) . \quad$ To see that $(3) \Rightarrow(2)$, let $A$ be a divisorial ideal of $R$. Then by Proposition 2.4

$$
\operatorname{div} A=\sum n_{\alpha} \operatorname{div} M_{\alpha}
$$

where $\left\{M_{\alpha} \mid \alpha \in I\right\}$ is the collection of maximal divisorial ideals of $R$ and $n_{\alpha}=0$ almost everywhere on $I$. By (3), each $M_{\alpha}$ is principal and it follows that

$$
\operatorname{div} A=\sum n_{\alpha} \operatorname{div} M_{\alpha}=\operatorname{div} \Pi M_{\alpha}^{n_{\alpha}}
$$

is a principal divisor of $R$. Therefore, $C(R)$ is trivial and $(3) \Rightarrow(2)$.

$(2) \Rightarrow(4)$. Since each regular principal ideal of a ring is divisorial, it follows from Proposition 2.2 that every collection of regular principal ideals of a Krull ring has a maximal element. Hence, $R$ has property $(M)$. If $a$ and $b$ are regular elements of $R$, then $\overline{(a) \cap(b)} \subset(a)$ and $\overline{(a) \cap(b)} \subset(b)$. Thus $(a) \cap(b)$ is divisorial since $(a) \cap(b) \subset \overline{(a) \cap(b)}$, and it follows from the assumption that $C(R)$ is trivial that $(a) \cap(b)$ is principal. Therefore $(2) \Rightarrow(4)$.

$(5) \Leftrightarrow(3)$. Let $K$ be the total quotient ring of $R$ and let $M$ be a maximal divisorial ideal of $R$. Thus, if $S=\{a \in K \mid M \subset R a\}$, then $M=\bigcap\{R a \mid a \in S\}$. Therefore $M \subset R a \cap R \subset R$ for each $a \in S$. If $R a \cap R=R$ for each $a \in S$, then $R \subset \cap\{R a \mid a \in S\}=M$, a contradiction. Hence there exists $a \in S$ such that $M \subset R a \cap R$ which is properly contained in $R$. But $R a \cap R$ is a divisorial ideal of $R$, and by the maximality $M, M=R a \cap R$. Let $d$ be a regular element of $R$ such that $a d \in R$. Then $d M=R a d \cap R d$, and since $a d$ is regular, it follows that $d M$ is principal. Hence $M$ is principal and $(5) \Rightarrow(3)$. Conversely, since the intersection of two regular principal ideals is divisorial, from Proposition 2.4 it follows that the intersection of two regular principal ideals of $R$ is principal. Therefore $(5) \Leftrightarrow(3)$.

$(1) \Rightarrow(2)$. Since $R$ is a unique factorization ring, $R$ is a finite complete direct sum of unique factorization contains and special principal ideal rings. But each unique factorization domain is a Krull 
domain with trivial class group and a complete direct sum of special principal ideal rings is its own total quotient ring. Hence $R$ may be written as $R_{1} \oplus S$ where $R_{1}$ is a Krull ring and $S$ is its own total quotient ring. Therefore by Proposition 2.5, $R$ is a Krull ring.

Noting that the divisor class group of a finite complete direct sum is the complete direct sum of the divisor class groups of each summand, and that the divisor class group of $S$ is trivial, it follows that $C(R)$ is trivial. Therefore $(1) \Rightarrow(2)$, and the implications and equivalences in the statement of the proposition hold.

Of particular interest in Proposition 3.1 is the statement that (1) implies (2). However, it has not been shown whether the converse holds. If (2) did imply (1), then it would follow that each Krull ring with trivial class group is a finite complete direct sum of unique factorization domains and special ideal rings. This, in the author's opinion, is too strong a result. Accordingly, the proof or disproof that (2) implies (1) is left as an open problem.

\section{REFERENCES}

1. C. R. Fletcher, The structure of unique factorization rings, Proc. Cambridge Phil. Soc., 67 (1970), 535-540.

2. M. D. Larsen and P. McCarthy, Multiplicative Theory of Ideals, Academic Press, New York, 1971.

3. O. Zariski and P. Samuel, Commutative Algebra, Vol. 1, Van Nostrand, Princeton, N.J., 1958.

Received February 16, 1979.

Central Missouri State University

WARRENSBURG, MO 64093 


\section{PACIFIC JOURNAL OF MATHEMATICS}

\section{EDITORS}

DONALD BABBITT (Managing Editor)

University of Galifornia

Los Angeles, California 90024

Hugo RossI

University of Utah

Salt Lake City, UT 84112

C. C. MoOre AND ANDrew OGG

University of California

Berkeley, CA 94720

\section{J. DugundjI}

Department of Mathematics University of Southern California

Los Angeles, California 90007

R. Finn and J. Milgram

Stanford University

Stanford, California 94305

ASSOCIATE EDITORS

E. F. Beckenbachi

B. H. NeumanN

F. WOLF

K. YOSHIDA

\section{SUPPORTING INSTITUTIONS}

UNIVERSITY OF BRITISH COLUMBIA

CALIFORNIA INSTITUTE OF TECHNOLOGY

UNIVERSITY OF CALIFORNIA

MONTANA STATE UNIVERSITY

UNIVERSITY OF NEVADA, RENO

NEW MEXICO STATE UNIVERSITY

OREGON STATE UNIVERSITY

UNIVERSITY OF OREGON
UNIVERSITY OF SOUTHERN CALIFONIA

STANFORD UNIVERSITY

UNIVERSITY OF HAWAII

UNIVERSITY OF TOKYO

UNIVERSITY OF UTAH

WASHINGTON STATE UNIVERSITY

UNIVERSITY OF WASHINGTON

Printed in Japan by International Academic Printing Co., Ltd., Tokyo, Japan 


\section{Pacific Journal of Mathematics \\ Vol. 89 , No. 1 \\ May, 1980}

David Bressoud, A note on gap-frequency partitions ................ 1

John David Brillhart, A double inversion formula ................. 7

Frank Richard Deutsch, Günther Nürnberger and Ivan Singer, Weak

Chebyshev subspaces and alternation .......................

Edward Richard Fadell, The relationship between Ljusternik-Schnirelman

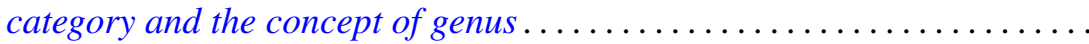

Harriet Jane Fell, On the zeros of convex combinations of polynomials.......

John Albert Fridy, An addendum to: "Tauberian theorems via block

dominated matrices" ..................................

Andrzej Granas, Ronald Bernard Guenther and John Walter Lee, Applications of topological transversality to differential equations. I. Some nonlinear

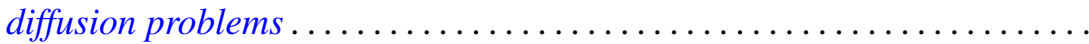

David E. Handelman and G. Renault, Actions of finite groups on self-injective

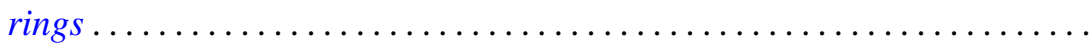

Michael Frank Hutchinson, Local $\Lambda$ sets for profinite groups .............

Arnold Samuel Kas, On the handlebody decomposition associated to a

Lefschetz fibration...

Hans Keller, On the lattice of all closed subspaces of a Hermitian space.....

P. S. Kenderov, Dense strong continuity of pointwise continuous

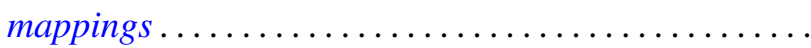

Robert Edward Kennedy, Krull rings.................

Jean Ann Larson, Richard Joseph Laver and George Frank McNulty,

Square-free and cube-free colorings of the ordinals ...

Viktor Losert and Harald Rindler, Cyclic vectors for $L^{p}(G)$

John Rowlay Martin and Edward D. Tymchatyn, Fixed point sets of

1-dimensional Peano continua...

Augusto Nobile, On equisingular families of isolated singularities ...

Kenneth Joseph Prevot, Imbedding smooth involutions in trivial bundles ...

Thomas Munro Price, Spanning surfaces for projective planes in four space.

Dave Riffelmacher, Sweedler's two-cocycles and Hochschild cohomology....

Niels Schwartz, Archimedean lattice-ordered fields that are algebraic over

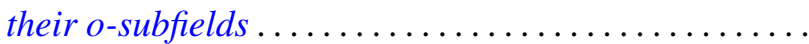

Chao-Liang Shen, A note on the automorphism groups of simple dimension groups.

Kenneth Barry Stolarsky, Mapping properties, growth, and uniqueness of

Vieta (infinite cosine) products ...

Warren James Wong, Maps on simple algebras preserving zero products. I.

The associative case ............................. 